Research Paper

\title{
NCOA4-RET fusion in colorectal cancer: Therapeutic challenge using patient-derived tumor cell lines
}

\author{
Sun Young Kim ${ }^{1 *}$, Seiyoon Oh $\mathrm{Oh}^{2 *}$, Kyung Kim ${ }^{1}$, Jeeyun Lee ${ }^{1}$, SoYoung Kang 3 , Kyoung-Mee Kim³, \\ WooYong Lee ${ }^{4}$, Seung Tae Kim ${ }^{1 凶}$, and Dohyun Nam Nam ${ }^{5 凶}$ \\ 1. Division of Hematology-Oncology, Department of Medicine, Samsung Medical Center, Sungkyunkwan University School of Medicine, Seoul, Korea \\ 2. Department of Human Biology, Health and Society, College of Human Ecology, Cornell University, Ithaca, NY 14850, USA \\ 3. Department of Pathology and Translational Genomics, Samsung Medical Center, Sungkyunkwan University School of Medicine, Seoul, Korea \\ 4. Department of Surgery, Samsung Medical Center, Sungkyunkwan University School of Medicine, Seoul, Korea \\ 5. Department of Neurosurgery, Samsung Medical Center, Sungkyunkwan University School of Medicine, Seoul, Korea \\ * Equally contributed
}

$\triangle$ Corresponding author: Seung Tae Kim, M.D., Division of Hematology/Oncology, Department of Medicine, Samsung Medical Center, Sungkyunkwan University School of Medicine, 81 Irwon-ro, Gangnam-gu, Seoul 06351, Korea. Tel: +82-2-3410-0297; Fax: +82-2-3410-1754; E-mail: seungtae1.kim@samsung.com \& Dohyun Nam, M.D., Department of Neurosurgery, Samsung Medical Center, Sungkyunkwan University School of Medicine, 81 Irwon-ro, Gangnam-gu, Seoul 06351, Korea. Tel: +82-2-3410-3497; Fax: +82-2-3410-1754; E-mail: dhns.nam@samsung.com

(c) Ivyspring International Publisher. This is an open access article distributed under the terms of the Creative Commons Attribution (CC BY-NC) license (https://creativecommons.org/licenses/by-nc/4.0/). See http://ivyspring.com/terms for full terms and conditions.

Received: 2018.03.23; Accepted: 2018.06.09; Published: 2018.07.30

\begin{abstract}
The RET fusion is considered as the potential novel target in solid tumors. However, RET fusion is not well yet identified in colorectal cancer (CRC), and the effect of RET kinase inhibitor is also not evaluated in CRC with RET fusion. We established patient-derived tumor cells (PDCs) with RET fusion from recurrent brain metastatic lesion that newly appeared during the surveillance for stage III CRC patient. To investigate therapeutic options to CRC patient with a RET fusion, we performed cell viability assays using the PDCs. NCOA4-RET fusion was detected by FusionPlex using the resected brain metastatic tissue of CRC patient with solitary brain metastasis and then reconfirmed by fluorescence in situ hybridization (FISH) test. We also confirmed the RET fusions by a qPCR in matched PDCs. We tested whether the PDCs from RET fusion colon cancer were sensitive to carbozantinib, sorafenib, vandetanib, and PD0331992. Cell viability assays showed that carbozantinib, sorafenib, and PD0332991 did not suppress cell viability. Only, vandetanib revealed the significant inhibitory effect in MTT proliferation assay. Next, we analyzed regulation of targeted downstream pathways upon exposure to vandetanib by immunoblot assay. In colon cancer PDCs with NCOA4-RET fusion, vandetanib potently inhibited AKT and ERK phosphorylation. This study shows that vandetanib might be one of useful treatment strategies for CRC patient with NCOA4-RET fusion. Therefore, inhibition of the RET kinase is a promising targeted therapy for cancer patients whose tumors harbor a RET rearrangement.
\end{abstract}

Key words: Colorectal cancer, RET fusion, vandetanib

\section{Introduction}

An improved understanding of the underlying molecular pathology of colorectal cancer (CRC) has enabled tailored treatment regimens and helped to optimize outcomes. There have been recent and rapid advances in the development of agents targeting components of receptor tyrosine kinase signaling cascades for use in cancer therapy.[1-3] A monoclonal antibody $(\mathrm{mAb})$ against epidermal growth factor receptor (EGFR), referred to as cetuximab, is a product of this movement, and has been implemented to treat selected metastatic CRC patients. Only the absence of an oncogenic KRAS and/or NRAS mutation has been found to predict the clinical benefit from anti-EGFR therapy in metastatic CRC. However, unfortunately, except anti-EGFR therapies, genomic characterization of CRC has not been translated into effective target matched therapeutic strategies.

RET is a transmembrane tyrosine kinase that functions as the receptor for growth factors of the glial derived neurotrophic factor family.[4] Abnormal 
chromosomal rearrangements cause the inframe fusion of tyrosine kinase domain of the RET gene fusions can lead to the expression of oncogenic driver fusion kinase.[5] RET fusion kinase occur in nearly one third of papillary thyroid cancer and about $2 \%$ of lung adenocarcinoma.[6] More than 13 fusion partner genes of RET have been reported until now. In lung adenocarcinoma and thyroid carcinoma with RET fusion, some RET kinase inhibitors such as sunitinib, sorafenib, carbozantinib and vandetanib showed anti-tumor activity in preclinical models and patients with lung cancer.[7-10] However, RET fusion is not well yet identified in CRC, and the effect of RET kinase inhibitor is also not evaluated in CRC with RET fusion.

We established patient-derived cells (PDC) from CRC patient with a RET fusion that had the brain metastasis. We used these PDCs to investigate useful therapeutic options to realize the precision medicine in CRC with RET fusion.

\section{Patients and Methods}

Patient-derived tumor cells. With informed consent, tumor samples were obtained from newly appeared single brain lesion of a patient of stage III CRC who had received curative surgery followed by post-operative chemotherapy. Collected tissue from brain lesion was minced and dissociated by enzymatic methods. The patient-derived cells (PDCs) from a metastatic brain lesion were cultured in RPMI 1640 supplemented with $10 \%$ fetal bovine serum (FBS; Gibco BRL, Paisley, UK), 1\% antibiotic/anti-mycotic solution (Gibco BRL, Paisley, UK), $0.5 \mu \mathrm{g} / \mathrm{ml}$ of hydrocortisone (Sigma Aldrich), $5 \mu \mathrm{g} / \mathrm{ml}$ of insulin (PeproTech, Rocky Hill, NJ, USA), 5 ng of EGF and 2.5 ng of FGF (PeproTech, Rocky Hill, NJ, USA). The medium was changed every 3 days, and cells were maintained at $37^{\circ} \mathrm{C}$ in a humidified $5 \% \mathrm{CO}_{2}$ incubator. PDCs were passaged using TrypLE Express (Gibco BRL, Paisley, UK) to detach cells when they reached 80-90\% confluence.

Targeted sequencing. Formalin-fixed paraffin embedded (FFPE) samples containing $>40 \%$ tumor cellularity were dissected under a microscope from $4-\mu \mathrm{m}$ thick unstained sections (10 to 20 slides) or from fresh frozen tissues by comparison with a hematoxylin and eosin-stained slide. RNA extracted from FFPE and synthesized the cDNA libraries and then enriched the target that can be used to create libraries for next generation sequencing using FusionPlex CTL kit (ArcherDX, Boulder, CO, USA). The sequencing data were analyzed using the Archer Analyzer Platform to detect the fusion and mutation.

RET translocation with fluorescence in situ hybridization (FISH). FISH was performed using the
ZytoLight $^{\mathrm{R}}$ SPEC RET Dual Color Break Apart Probe Kit (ZytoVision, Bremerhaven, GERMANY) for RET. This probe is designed to detect translocation involving the chromosomal region 10q11.21 harboring the RET (rearranged during transfection protooncogene) gene. It is a mixture of two direct labeled probes hybridizing to the $10 \mathrm{q} 11.21$ band. The orange fluorochrome direct labeled probe hybridizes proximal to the RET gene, the green fluorochrome direct labeled probe hybridizes distal to that gene.

Quantitative real-time reverse transcriptionpolymerase chain reaction. Total RNA was isolated from cells using the Qiagen RNeasy Mini Kit protocol (Qiagen, Valencia, CA, USA) according to the manufacturers' recommendations. Total RNA from each sample was reverse transcribed with the SuperScript ${ }^{\circledR I I I}$ First-Strand Synthesis System using Oligo(dT) 20 primer (Invitrogen Corp, Carlsbad, California, USA). All qPCR reactions were performed with a 7500 Fast Real-Time PCR System (Applied Biosystems, Foster City, CA), using NCOA4-RET fusion primer Forward: 5' - TAAAGGTGGTGGGCAA AAACAA-3' Reverse: 5'- CGGACGCGACGGAC AC-3', and GAPDH (Forward:5'-ACAACTTTGGTA TCGTGGG-3' Reverse: 5' - GCCATCACGCCACAGT TTC-3') was used as the housekeeping gene for relative quantification. All genes were run in triplicate to allow for the assessment of technical variability.

Cell treatment and viability assay. After pathologic confirmation, cells were seeded at a density of 1-2 × $10^{6}$ cells/10-mm dish for immunoblot analysis or 5,000 cells/well in 96-well plates for cell viability assays and treated for 3-5 days with various concentrations of drugs such as carbozantinib, sorafenib, vandetanib, and PD0331992 as indicated. Inhibition of cell proliferation was determined using Cell Titer Glo (Promega, Madison, WI, USA) according to the manufacturer's protocol.

Immunoblot Analysis. Total proteins from PDCs were isolated cOmplete Lysis-M (Roche) containing a protease inhibitor cocktail (Roche, Mannheim, Germany) and phosphatase inhibitor cocktail (Roche), and protein concentrations were determined according to Bradford procedure using a Quick Start Bradford Protein Assay (Bio-Rad, Hercules, CA, USA). Thirty $\mu \mathrm{g}$ of proteins were subjected to $10 \%$ SDS-polyacrylamide gel electrophoresis, and electro-transferred onto nitocellulose membranes. The membranes were blocked with $5 \%$ nonfat dry milk in Tris-buffered saline containing $0.1 \% \mathrm{v} / \mathrm{v}$ Tween 20 , and probed overnight at $4{ }^{\circ} \mathrm{C}$ with a Specific antibodies: RET from Abcam (Cambridge, UK), pAkt (Ser473), Akt(C67E7), pERK1/2 (Thr202/Tyr204), ERK1/2 (Thr202/Tyr204) from Cell Signaling Technology (Beverly, MA, USA), 
and beta actin from Sigma Aldrich. Horseradish peroxidase-conjugated anti-rabbit or mouse IgG (Vector, Burlingame, CA, USA) were used as a secondary antibody, and signals were detected by chemiluminescence using ECL Western Blotting Substrate (Thermo Scientific, Rockford, IL, USA) and visualized using LAS-4000 (Fujifilm, Tokyo, Japan).

\section{Results}

Patient. A 63-year-old man initially presented with stage III, rectosigmoid colon cancer in 2013. He underwent lower anterior resection for the primary lesion and then received eight cycles of adjuvant XELOX (capecitabine and oxaliplatin) chemotherapy.
During regular surveillance, at 2015, the patient suffered from an abrupt headache. Brain imaging for the patient revealed a single brain mass. Patient received a curative intent brain surgery for the single brain mass suggesting a malignant primary brain tumor (Fig. 1). After pathologic confirmation for tumor, we generated PDCs from the patient. Finally, this resected brain lesion was confirmed in a metastatic recurrent colon cancer. NCOA4-RET fusion was detected by FusionPlex using the resected tumor sample and then reconfirmed by fluorescence in situ hybridization (FISH) test (Fig. 2). We also confirmed the RET fusions by a qPCR in matched PDCs (Fig. 3).
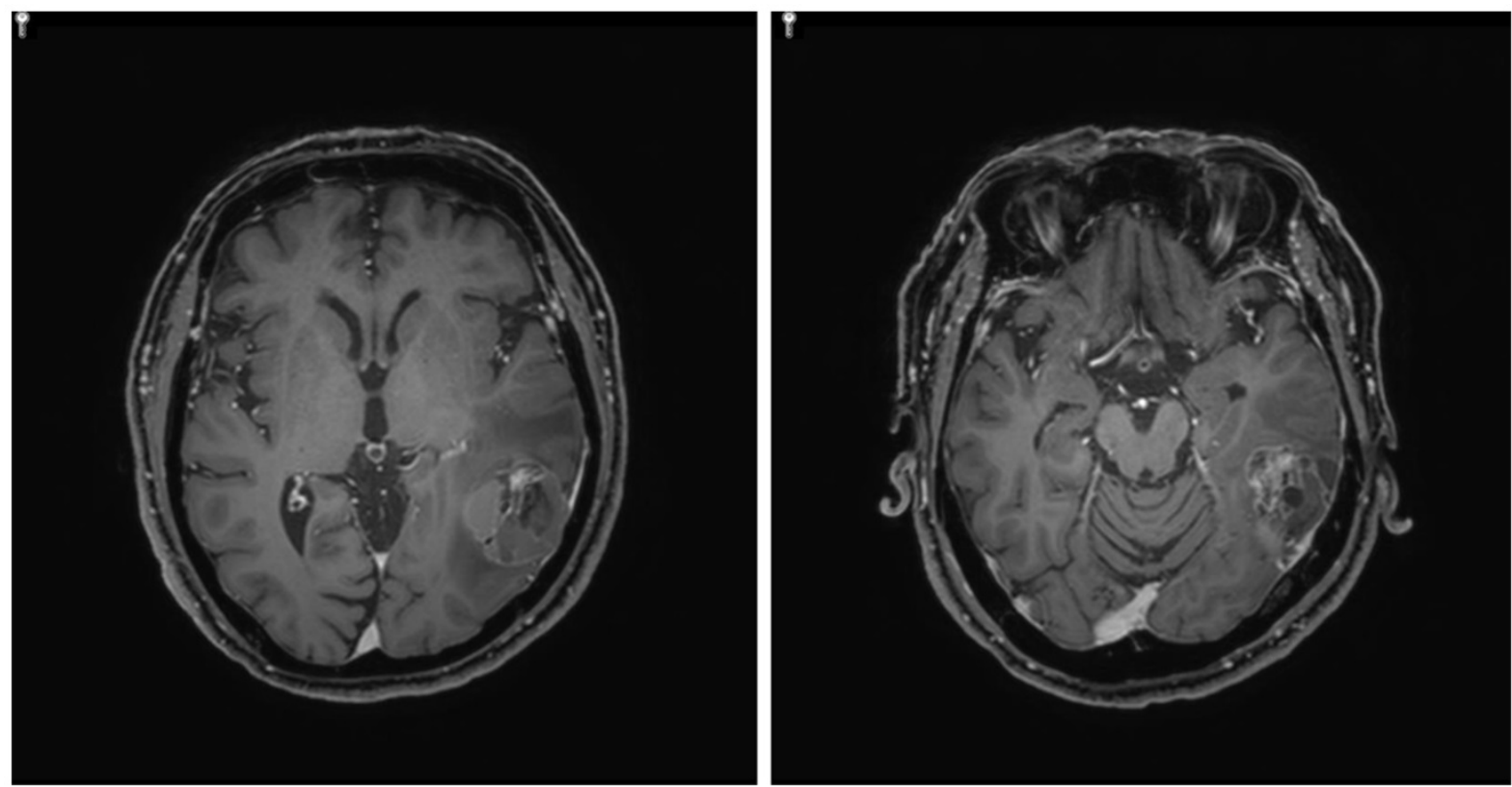

Figure 1. Single brain mass in magnetic resonance imaging (MRI)
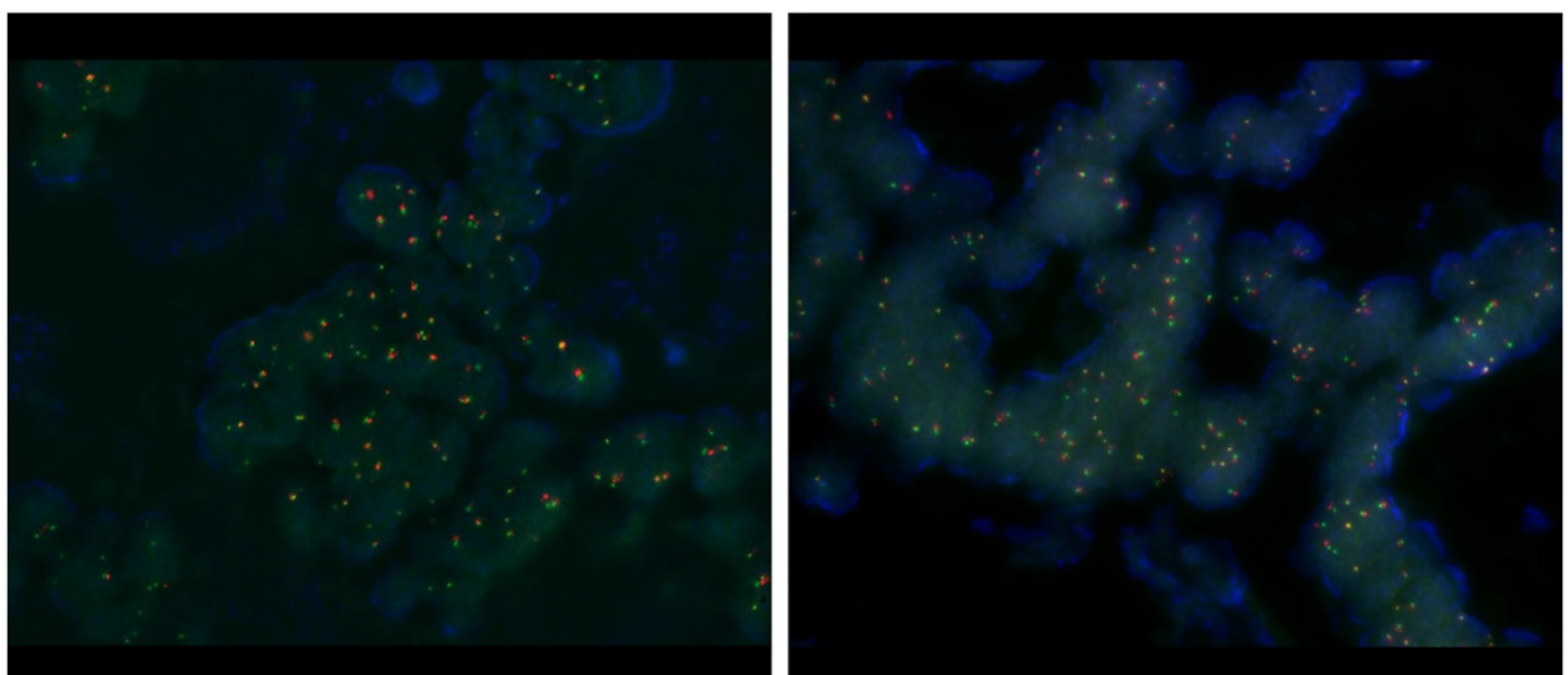

Figure 2. Fluorescence in situ hybridization (FISH) test. 
(a)

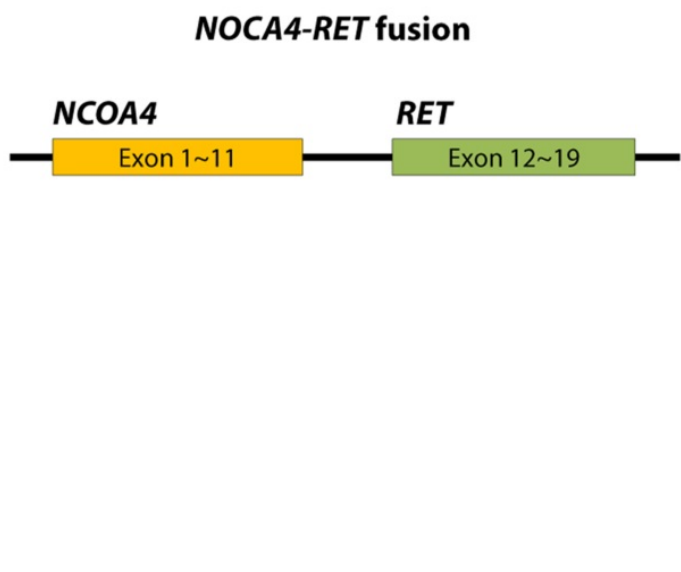

(b)

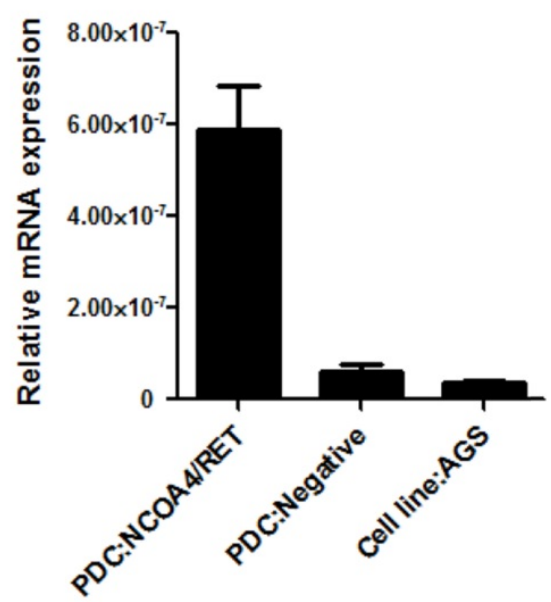

Figure 3. Confirmation of the RET fusions by a QPCR in matched PDCs (a) and (b).

(a)

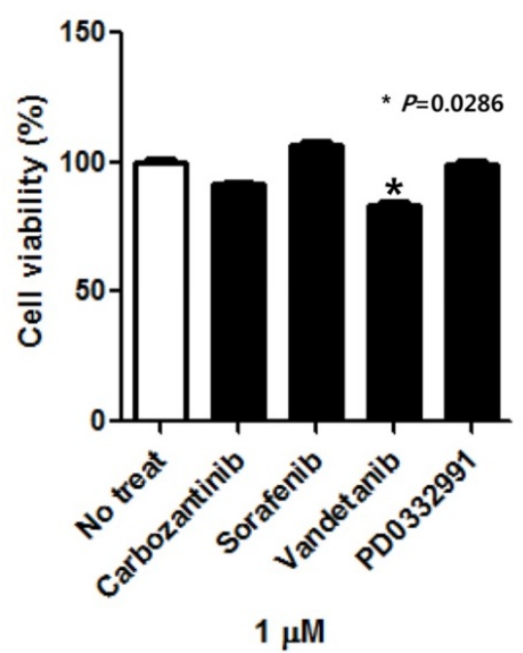

(b)

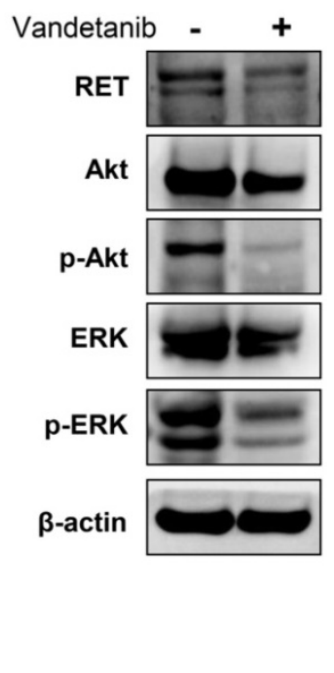

Figure 4. A cell viability assay in patient-derived tumor cells (PDCs) with RET fusion (a) and targeted downstream pathways upon exposure to vandetanib by immunoblot assay (b).

Cell viability assay using patient-derived cells. To investigate therapeutic options applicable to CRC with RET fusion; we performed a cell viability assay using the established NCOA4-RET fusion CRC PDC line. We tested whether the PDCs from RET fusion colon cancer were sensitive to carbozantinib, sorafenib, vandetanib, and PD0331992 that were previously reported as effective agents to RET fusion tumor cells in preclinical model or other types of solid cancers. Cell viability assays showed that carbozantinib, sorafenib, and PD0332991 did not suppress cell viability. Only, vandetanib revealed the significant inhibitory effect in MTT proliferation assay (Fig. 4A).

Immunoblot assay to vandetanib using patient-derived cells. Next, we analyzed regulation of targeted downstream pathways upon exposure to vandetanib by immunoblot assay. In colon cancer
PDCs with NCOA4-RET fusion, vandetanib potently inhibited AKT and ERK phosphorylation (Fig. 4B). These immunoblot findings were well concordant to the inhibitory effect of vandetanib.

\section{Discussion}

The RET fusion is considered as the potential novel target in solid tumors. Interestingly, RET fusions are mutually exclusive with activating mutations in EGFR, HER2, BRAF, and KRAS as well as EML4-ALK and ROS1 rearrangement in non-small cell lung cancer (NSCLC).[11, 12] This finding is consistent to CRC. Anne-France et al reported that in CRC, RET fusion did not overlap with other known druggable mutation such as KRAS, BRAF, EGFR, and PIK3CA, and oncogenic fusion kinases involving ALK, ROS1 and NTRK.[13] Considering these findings, subpopulation with RET fusion in solid 
tumors including CRC might need the specific treatment strategy in the precision era. In the present study, CRC PDCs with NCOA4-RET fusion were sensitive to vandetanib, with downregulation of the downstream pathway involving AKT and ERK phosphorylation. These findings suggested that vandetanib might be a novel treatment strategy for CRC patients with NCOA4-RET fusion.

Vandetanib is orally active small molecule receptor tyrosine kinase inhibitor vascular endothelial growth factor receptor (VEFR), epidermal growth factor receptor 2 (EGFR2), EGFR and RET.[9, 14] Vandetanib showed anti-tumor activity in cell lines with RET fusions and has also demonstrated the efficacy in the treatment of medullary thyroid cancer. In addition to vandetanib, carbozantinib, sorafenib, and sunitinib have also been shown pre-clinically to inhibit RET kinase activity.[7-9, 14] However, these agents have not been evaluated in CRC until now. In this study, we evaluated the anti-tumor effect of known various RET inhibitor in CRC-PDCs with RET fusion. Although only vandetanib had a significant antitumor effect to CRC-PDC with RET fusion in our study, this finding must be interpreted with caution because this study was for only single case of a CRC-PDC with NCOA4-RET fusion. In recent clinical trials for pretreated patients with RET rearranged NSCLC, vandetanib, and carbozantinib, showed the antitumor activity and tolerable safety[15-17]. The results of these trials might help to clarify the usefulness of RET inhibitors and choice the more potent one among various RET inhibitors.

RET fusion occur in approximately $1 \%$ to $2 \%$ of unselcted NSCLC and nearly one-third of papillary thyroid cancer.[5, 18] In CRC, recent western study reported the frequeuncy of $0.2 \%, 6$ of 3117 CRC patients.[13] We have been conducting the comprehensive targeted sequencing in gastrointestinal cancer patients as clinical sequencing program. Among metastatic refractory CRC patients included in this program, we found two CRC patients with RET fusion. One patient had NCOA4-RET fusion, mentioned in the present study and the other patient had KIF5B-RET fusion. The frequency of RET fusion in our CRC cohort was $1.6 \%$ (2 of 120). The frequency between our cohort and previous western cohort was discordant. This may be caused by heterogeneous patients' population, small sample size and ethnic difference. In the future, international molecular cohort study including RET fusion in CRC must be needed to establish the optimal treatment strategy for heterogeneous CRC in precision medicine era.

Accurate prediction of anti-tumor effects of molecularly targeted agents before clinical trial design and implementation in cancer patients is essential to realize the goal of precision medicine. Ideal preclinical models should closely resemble the actual tumors in terms of genomic profiles and drug response. Recently, patient-derived tumor cells have been suggested as an alternative preclinical model for use as a prediction system for preclinical drug testing. Our group previously demonstrated the usefulness of our PDC system as a promising model for preclinical experiments in various cancer types including CRC.[19] In the present study, we also successfully established PDCs from a metastatic brain lesion that newly appeared in a patient with stage III CRC. Although CRC with NCOA4-RET fusion is very rare, it is important to establish a precise treatment strategy based on the genomic profile. From this perspective, our successfully established PDCs with NCOA4-RET fusion from a CRC patient with NCOA4-RET fusion could be considered important preparation for preclinical research.

This study shows that vandetanib might be one of useful treatment strategies for CRC patient with NCOA4-RET fusion. Therefore, inhibition of the RET kinase is a promising targeted therapy for cancer patients whose tumors harbor a RET rearrangement.

\section{Acknowledgements}

This work was supported by a grant from the Korean Health Technology R\&D Project, Ministry of Health \& Welfare, Republic of Korea (HI14C3418). Support was also provided by a grant from Basic Science Research Program through the National Research Foundation of Korea(NRF) funded by the Ministry of Education (2016R1A6A3A11932444)

\section{Competing Interests}

The authors have declared that no competing interest exists.

\section{References}

1. Cunningham D, Humblet $Y$, Siena S, et al. Cetuximab monotherapy and cetuximab plus irinotecan in irinotecan-refractory metastatic colorectal cancer. N Engl J Med. 2004; 351: 337-345.

2. Hurwitz H, Fehrenbacher L, Novotny W, et al. Bevacizumab plus irinotecan, fluorouracil, and leucovorin for metastatic colorectal cancer. N Engl J Med. 2004; 350: 2335-2342.

3. Mayer RJ. Targeted therapy for advanced colorectal cancer--more is not always better. N Engl J Med. 2009; 360: 623-625.

4. Jhiang SM. The RET proto-oncogene in human cancers. Oncogene. 2000; 19: 5590-5597.

5. Santoro M, Melillo RM, Fusco A. RET/PTC activation in papillary thyroid carcinoma: European Journal of Endocrinology Prize Lecture. Eur J Endocrinol. 2006; 155: 645-653.

6. Mulligan LM. RET revisited: expanding the oncogenic portfolio. Nat Rev Cancer. 2014; 14: 173-186.

7. Drilon A, Wang L, Hasanovic A, et al. Response to Cabozantinib in patients with RET fusion-positive lung adenocarcinomas. Cancer Discov. 2013; 3: 630-635.

8. Goodwin PJ, Ennis M, Pritchard KI, et al. Insulin- and obesity-related variables in early-stage breast cancer: correlations and time course of prognostic associations. J Clin Oncol. 2012; 30: 164-171. 
9. Lipson D, Capelletti M, Yelensky R, et al. Identification of new ALK and RET gene fusions from colorectal and lung cancer biopsies. Nat Med. 2012; 18: 382-384.

10. Carlomagno F, Anaganti S, Guida T, et al. BAY 43-9006 inhibition of oncogenic RET mutants. J Natl Cancer Inst. 2006; 98: 326-334.

11. Eng C. RET proto-oncogene in the development of human cancer. J Clin Oncol. 1999; 17: 380-393.

12. Ju YS, Lee WC, Shin JY, et al. A transforming KIF5B and RET gene fusion in lung adenocarcinoma revealed from whole-genome and transcriptome sequencing. Genome Res. 2012; 22: 436-445.

13. Le Rolle AF, Klempner SJ, Garrett CR, et al. Identification and characterization of RET fusions in advanced colorectal cancer. Oncotarget. 2015; 6: 28929-28937.

14. Carlomagno F, Vitagliano D, Guida T, et al. ZD6474, an orally available inhibitor of KDR tyrosine kinase activity, efficiently blocks oncogenic RET kinases. Cancer Res. 2002; 62: 7284-7290.

15. Drilon A, Rekhtman N, Arcila M, et al. Cabozantinib in patients with advanced RET-rearranged non-small-cell lung cancer: an open-label, single-centre, phase 2, single-arm trial. Lancet Oncol. 2016; 17: 1653-1660.

16. Lee $\mathrm{SH}$, Lee $\mathrm{JK}, \mathrm{Ahn} \mathrm{MJ}$, et al. Vandetanib in pretreated patients with advanced non-small cell lung cancer-harboring RET rearrangement: a phase II clinical trial. Ann Oncol. 2017; 28: 292-297.

17. Yoh K, Seto T, Satouchi $M$, et al. Vandetanib in patients with previously treated RET-rearranged advanced non-small-cell lung cancer (LURET): an open-label, multicentre phase 2 trial. Lancet Respir Med. 2017; 5: 42-50.

18. Takeuchi K, Soda M, Togashi $\mathrm{Y}$, et al. RET, ROS1 and ALK fusions in lung cancer. Nat Med. 2012; 18: 378-381.

19. Song HN, Lee C, Kim ST, et al. Molecular characterization of colorectal cancer patients and concomitant patient-derived tumor cell establishment. Oncotarget. 2016; 7: 19610-19619. 